\title{
Schlechte Nachrichten:
}

\section{»Fake News« in Politik und Öffentlichkeit}

\author{
Romy Jaster \& David Lanius ${ }^{1}$
}

Abstract

Das Funktionieren moderner Demokratien hängt von der Informiertheit der Öffentlichkeit ab. Durch den Erfolg von »Fake News" und postfaktischer Politik ist diese jedoch in Gefahr, zumal parallele Öffentlichkeiten zunehmend sogenannte alternative analoge und digitale Medienangebote nutzen. In diesem Beitrag untersuchen wir, wie sich »Fake News" verbreiten und Einfluss auf Öffentlichkeit und Politik gewinnen. Dazu analysieren wir das Zusammenspiel einer Reihe kognitiver Verzerrungen mit der Funktionsweise sozialer Medien sowie die strukturellen Anreize, die der digitalisierte Medienkapitalismus setzt. Beides spielt der Verbreitung von "Fake News" in die Hände, was einige politische Akteure auszunutzen wissen. »Fake News" werden in diesem Fall als Propaganda eingesetzt. Wir unterscheiden vier Funktionen von »Fake News«-Propaganda: (1) die Täuschung der Öffentlichkeit, (2) die Stärkung von Gruppenidentitäten, (3) die Demonstration von Macht und (4) die Destabilisierung der politischen oder öffentlichen Ordnung.

\section{Einleitung}

Das Funktionieren moderner Demokratien hängt von der Informiertheit der Öffentlichkeit ab (Lippmann, 1922; Sproule, 2005). Durch den Erfolg von »Fake News « und postfaktischer Politik ist diese jedoch in Gefahr, zumal parallele Öffentlichkeiten zunehmend sogenannte alternative analoge und digitale Medienangebote nutzen (Benkler, Faris \& Roberts, 2018). Mit dem Brexit und der Präsidentschaft Donald Trumps als politische Konstanten der letzten zwei Jahre sowie dem zunehmenden Einfluss rechtspopulistischer Parteien und russischer Staatsmedien in immer mehr Ländern der Welt

1 Der Beitrag stammt in gleichen Teilen von beiden Autoren. 
haben »Fake News« unleugbar einen festen Platz in der Politik gefunden. Wie konnte es dazu kommen?

Im Folgenden geben wir eine Antwort auf diese Frage, indem wir untersuchen, wie sich »Fake News« verbreiten und Einfluss auf Öffentlichkeit und Politik gewinnen. Unsere Überlegungen bedienen sich philosophischer, aber auch psychologischer und kommunikationswissenschaftlicher Erkenntnisse. Im ersten Abschnitt geben wir eine knappe Definition von »Fake News«. Im zweiten Abschnitt analysieren wir das Zusammenspiel einer Reihe kognitiver Verzerrungen mit der Funktionsweise der sozialen Medien. Dabei gehen wir auch auf die strukturellen Anreize ein, die der digitalisierte Medienkapitalismus setzt. Beides spielt der Verbreitung von »Fake News« in die Hände. Im dritten Abschnitt zeigen wir, wie sich die Verbreitungsmechanismen von $»$ Fake News« politisch nutzen lassen. Wir unterscheiden vier Funktionen, mit denen politische Akteure »Fake News « als Propaganda einsetzen. »Fake News«-Propaganda wird eingesetzt, (1) um über Tatsachen zu täuschen, (2) um Gruppenidentitäten zu stärken, (3) um Macht zu demonstrieren und (4) um die politische oder öffentliche Ordnung zu destabilisieren.

\section{Was »Fake News« sind}

In jüngerer Zeit sind eine Reihe von Definitionen des »Fake News«-Begriffs vorgelegt worden, die jeweils unterschiedliche Aspekte des Phänomens ins Zentrum stellen (Dentith, 2017; Gelfert, 2018; Rini, 2017; Mukerji, 2018; Zimmermann \& Kohring, 2018). ${ }^{2}$ Unserer eigenen Analyse zufolge sind $»$ Fake News« Berichterstattungen, die in zwei spezifischen Hinsichten problematisch sind. Erstens vermitteln sie ein inkorrektes Bild der Wirklichkeit, da sie entweder falsch oder irreführend sind. Zweitens mangelt es ihren Verfassern an Wahrhaftigkeit, da diese entweder eine Täuschungsabsicht verfolgen oder der Wahrheit gleichgültig gegenüberstehen (Jaster \& Lanius, 2018, 2019). ${ }^{3}$ Wenn wir von »Fake News « sprechen, verwenden wir den Begriff gemäß dieser Definition.

Damit folgen wir nicht der Position, dass man im öffentlichen und wissenschaftlichen Diskurs besser auf die Rede von »Fake News« verzichten

2 Für einen systematischen Vergleich dieser Ansätze siehe Jaster \& Lanius (im Erscheinen b).

3 Ist ein Sprecher einer Behauptung der Wahrheit gegenüber gleichgültig, spricht man auch von Bullshit (Frankfurt 1986, Fallis \& Stokke 2017). Dazu mehr in Abschnitt 3 . 
solle. Eine gängige Kritik an Definitionsversuchen des »Fake News«-Begriffs macht geltend, der Ausdruck »Fake News « werde mit ganz unterschiedlichen Bedeutungen verwendet, denn er diene sowohl als Kennzeichnung für defizitäre Berichterstattung als auch als Mittel zur Diskreditierung politischer Gegner. Aufgrund dieser Doppelbelegung des Begriffs sei es nahezu unmöglich, »Fake News « zu definieren, und insgesamt besser, im öffentlichen und wissenschaftlichen Diskurs ganz auf den Begriff zu verzichten (Wardle \& Derakhshan, 2017, Habgood-Coote, 2018). ${ }^{4}$

Wir halten diese Überlegung für fehlgeleitet. Wenn Donald Trump beispielsweise die New York Times als »Fake News« bezeichnet, dann verwendet er »Fake News« nicht in einer anderen Bedeutung als eine Soziologin, die untersucht, welche Ursachen die Verbreitung von »Fake News« hat. In beiden Fällen sind mit »Fake News« Berichterstattungen gemeint, die einen Mangel an Wahrheit und einen Mangel an Wahrhaftigkeit aufweisen. Genau das ist es, was Donald Trump der New York Times vorwirft: falsche oder irreführende Berichte zu verbreiten, um das Publikum (beispielsweise über Trump und seine Administration) zu täuschen oder ohne sich überhaupt für die Wahrheit zu interessieren. Uneinigkeit mag darüber bestehen, auf wessen Berichterstattung diese Beschreibung tatsächlich zutrifft. Die Bedeutung von »Fake News« hingegen ist in beiden Kontexten dieselbe. Wir halten es für einen Vorzug der oben genannten Definition, dass sie sowohl dem in den Wissenschaften untersuchten Phänomen von »Fake News « als auch dem Einsatz des »Fake News«-Begriffs in politischen Grabenkämpfen gerecht wird.

\section{Wie sich »Fake News« verbreiten}

»Fake News« sind kein neues Phänomen. Falsche oder irreführende Berichterstattungen, die mit einer Täuschungsabsicht oder mit einer Haltung der Gleichgültigkeit gegenüber der Wahrheit verbreitet werden, finden sich in der Geschichte zuhauf (Keil \& Kellerhoff, 2017; McIntyre, 2018; Jaster \& Lanius, 2019). Dennoch ist es kein Zufall, dass »Fake News « im Zeitalter der Digitalisierung eine beispiellose Verbreitung erfahren. ${ }^{5}$

4 Man kann zudem sagen, dass der Ausdruck »Fake News« hochgradig kontrovers und unbestimmt ist, wie das Marwick \& Lewis (2017) tun, wenn sie »Fake News« als »contested term« bezeichnen. Aus unserer Sicht ist dies jedoch ein umso stärkerer Grund, den Begriff für den wissenschaftlichen Gebrauch eindeutig zu definieren und das Phänomen präzise zu beschreiben.

5 Siehe dazu Mathiesen (2019). 
Die grundlegende Dynamik der Rezeption und Verbreitung von »Fake News« erklärt sich durch die Gesetzmäßigkeiten der digitalen Aufmerksamkeitsökonomie. Wenn Informationen im Überfluss vorhanden sind, ist Aufmerksamkeit eine knappe Ressource. In Zeiten des Internets existieren mehr Informationen, als Konsumenten verarbeiten können. Da die Aufmerksamkeit der Konsumenten begrenzt ist, verbrauchen sie ihre Aufmerksamkeit für eine Information immer auf Kosten ihrer Aufmerksamkeit für andere Informationen. Je mehr Aufmerksamkeit eine Information auf sich zieht, desto höher ist daher ihr Wert (Simon, 1971; Davenport \& Beck, 2001).

Der Wert aufmerksamkeiterregender Meldungen kann in der Möglichkeit politischer Einflussnahme bestehen. Aber er lässt sich auch monetarisieren. Meldungen, die Aufmerksamkeit erregen, können genutzt werden, um Aufmerksamkeit auf weitere Informationen zu lenken. Dieses Prinzip zeigt sich auch im digitalen Werbemarkt: Wer es schafft, mit Inhalten Aufmerksamkeit zu erzeugen und Klicks zu generieren, kann Werbung schalten und sich die Weitergabe der bereits erzeugten Aufmerksamkeit von den Werbetreibenden bezahlen lassen (Sunstein, 2014, 2017).

»Fake News« haben in der Aufmerksamkeitsökonomie leichtes Spiel, weil sich die Wahrheit einer Nachricht nach aufmerksamkeitsökonomischen Maßstäben nicht unbedingt auszahlt. Menschen richten ihre Aufmerksamkeit besonders stark auf einfache Aussagen, Berichte über Ungewöhnliches (Sensationalismus), negative Botschaften (Negativitätsbias) und vertraut erscheinende Nachrichten (Vertrautheitsbias). ${ }^{6}$ Die Präferenz von Konsumenten für Einfaches, Ungewöhnliches, Negatives und Vertrautes können Produzenten von »Fake News« in einem Ausmaß bedienen, wie es redlich arbeitenden Journalisten nicht möglich ist. ${ }^{7}$ Nur wenn die Verpflichtung auf Sorgfalt und Wahrheit entfällt, können Informationen perfekt auf die Gesetzmäßigkeiten der Aufmerksamkeitsökonomie zugeschnitten werden.

Hinzu kommt, dass das mehrheitliche Nutzungsverhalten der sozialen Medien in einem Spannungsverhältnis zu Werten wie Wahrheitssuche und Erkenntnisgewinn steht. Das menschliche Gehirn ermöglicht zwei Arten

6 Die Aufmerksamkeit, die wir Nachrichten schenken, finden sich in den klassischen Nachrichtenwerten von Lippmann (1922) widergespiegelt, der zwischen Überraschung, Sensationalismus, Etablierung, Dauer, Struktur, Relevanz, Schaden, Nutzen, Prominenz und Nähe unterscheidet. Siehe auch Kepplinger (1998). Zum Negativitätsbias siehe Baumeister et al. (2001) und Hilbig (2009). Zum Vertrautheitsbias siehe Tversky \& Kahneman (1974).

7 Insbesondere Richtigstellungen von »Fake News« haben es aus diesem Grund schwer, in das öffentliche Bewusstsein vorzudringen. 
von Denkprozessen (Kahneman, 2012). System 1 ist das System für schnelles Denken. Das sind Denkprozesse, die schnell, intuitiv, automatisch, assoziativ und unbewusst ablaufen. System 2 ist das System für langsames Denken. Das sind Denkprozesse, die langsam, kalkulierend, gesteuert, analytisch und bewusst ablaufen. Eine der wichtigsten Erkenntnisse der jüngeren psychologischen Forschung ist: Biases brechen sich vor allem dann Bahn, wenn wir schnell, intuitiv und assoziativ - also mit System 1 - denken.

Wenn wir Inhalte der sozialen Medien verarbeiten, verwenden wir in der Regel System 1. Die sozialen Medien sind auf Bestätigung und Rückversicherung durch die soziale Gruppe ausgelegt. Werte wie Sorgfalt, Wahrheit, oder Wahrhaftigkeit stehen eher im Hintergrund. Das mehrheitliche Nutzungsverhalten der sozialen Medien ist somit dazu prädestiniert, »Fake News« ungeprüft weiterzuverbreiten. Wo schnelles Denken am Werk ist, haben »Fake News « besonders leichtes Spiel. ${ }^{8}$

Ein drittes Problem ist, dass die Nachteile unserer kognitiven Dispositionen im Zusammenspiel mit der Funktionsweise der sozialen Medien auf besonders problematische Weise in Erscheinung treten. Das Internet, und insbesondere Facebook, YouTube, WhatsApp, Instagram und Twitter, machen es Menschen besonders leicht, sich mit Gleichdenkenden zu umgeben (Graham, 2015; Bakshy, Messing \& Adamic, 2015). Das führt zu Echokammern, in denen bestimmte Kaskadenphänomene besonders schnell in Gang kommen können. In diesem Setting können »Fake News« florieren und Gruppenpolarisationsprozesse befeuern. Im Folgenden erläutern wir diese Dynamiken.

Einmal gestreute »Fake News« treffen bei einigen Menschen auf eine passend motivierte Wahrnehmung (Kunda, 1990; Kahan, 2013). Informationen, die mit unserem bestehenden Überzeugungssystem konfligieren, erzeugen kognitive Dissonanz: einen mentalen Zustand, der häufig als psychologischer Stress beschrieben wird. Kognitive Dissonanz wird unter anderem dann empfunden, wenn Menschen mit Evidenzen konfrontiert werden, die mit ihrem bestehenden Überzeugungs- oder Wertesystem konfligieren (Festinger, 2012). Kognitive Dissonanz ist unangenehm und wird daher vermieden.

Ein Resultat dieser Vermeidungstendenz ist der Bestätigungsfehler: Menschen neigen dazu, nach Informationen Ausschau zu halten, die ihre bestehenden Überzeugungen bestätigen (Nickerson, 1998; Oswald \&

8 Siehe Bronstein et al. (2018) sowie Pennycook \& Rand (2018). Aus demselben Grund haben es Richtigstellungen besonders schwer, die ihnen gebührende Aufmerksamkeit zu erzielen (Nyhan \& Reifler, 2010). 
Grosjean, 2004). Gegenläufigen Informationen messen sie weniger Plausibilität und Relevanz bei, und sie vergessen sie schneller wieder (Nickerson, 1998; Oswald \& Grosjean, 2004). Für »Fake News« bedeutet das: Entspricht eine solche Meldung dem Weltbild einer Person, wird der Nachricht von vornherein eine erhöhte Plausibilität beigemessen und seltener nach Informationen gesucht, die der Nachricht zuwiderläuft. Angesichts der Unterschiedlichkeit bestehender Überzeugungssysteme erklärt der Bestätigungsfehler, warum manche Menschen »Fake News« überzeugend finden, die andere Menschen für vollkommen unplausibel halten.

Die Vermeidung kognitiver Dissonanz und der Bestätigungsfehler führen dazu, dass Menschen sich in Echokammern organisieren (Sunstein, 2009, 2017). Echokammern sind dem Oxford English Dictionary zufolge soziale Räume, in denen Menschen nur solchen Überzeugungen oder Meinungen begegnen, die mit ihren eigenen zusammenfallen, so dass existierende Sichtweisen verstärkt und alternative Vorstellungen nicht erwogen werden. ${ }^{9}$ Korrigierende Einflüsse auf das Überzeugungssystem der Beteiligten bleiben in Echokammern aus.

Das Internet und insbesondere die Funktionsweise der sozialen Medien begünstigen die Tendenz, sich in Echokammern zurückzuziehen (Garrett, 2009; Gentzkow \& Shapiro, 2011; Bakshy, Messing \& Adamic, 2015). Die Meinungen der Nutzer sind in den sozialen Medien, insbesondere auf Twitter und Facebook, besonders sichtbar. Aufgrund der schieren Menge der online vernetzten Menschen findet sich für fast jede These eine Person, die sie glaubwürdig findet. ${ }^{10}$ Darüber hinaus gehört die gegenseitige Bestätigung in Form von Shares, Likes und Kommentaren zu den grundlegenden Funktionen sozialer Netzwerke. Man muss also nicht der Theorie von Filterblasen anhängen, um den Zusammenhang zwischen den sozialen Medien und der Ausbildung von Echokammern zu erklären. ${ }^{11}$

In Echokammern können besonders leicht Kaskadenphänomene auftreten. Eine sogenannte Informationskaskade liegt vor, wenn eine Meldung allein deshalb mehr Glaubwürdigkeit gewinnt, weil immer mehr Menschen die Meldung für wahr halten (Bikhchandani, Hirshleifer \& Welch, 1998). Informationskaskaden beruhen auf einer prinzipiell nützlichen Methode des

9 Eine ähnliche Definition schlägt Baumgaertner (2014) vor. Nguyen (2018) spricht stattdessen von »epistemic bubbles« und reserviert »Echokammern« für Räume, in denen gegenläufige Information aktiv unterdrückt wird.

10 Für eine Metastudie zur Verbreitung von Verschwörungstheorien in den USA siehe Oliver \& Wood (2014).

11 Die Theorie der Filterblasen stammt von Pariser (2011). Parisers Thesen sind umstritten und gelten als schlecht belegt. Siehe zum Beispiel Zuiderveen Borgesius et al. (2016). 
Erkenntnisgewinns. Wenn andere eine Information für wahr halten, ist das für uns in der Regel ein prima-facie-Grund, die Information ebenfalls für wahr zu halten. ${ }^{12}$ Wenn wir ein allgemeines Streben nach der Erkenntnis des Wahren unterstellen und davon ausgehen, dass unsere eigenen Erkenntnisfähigkeiten diejenigen der anderen nicht maßgeblich übersteigen, sollte es uns skeptisch machen, wenn andere unsere Meinungen nicht teilen, und es sollte uns in unseren Meinungen bestätigen, wenn sie es tun.

Problematisch wird es, wenn sich dieser Mechanismus verselbstständigt und Menschen sich gegenseitig in ihren Überzeugungen bestätigen, ohne dass jemand von ihnen unabhängige Gründe für die Überzeugung hätte. In einem solchen Fall spricht man von einer Informationskaskade. Die zugrundeliegende Dynamik setzt nicht voraus, dass die Beteiligten irrational sind. Eine Informationskaskade kann auch dann eintreten, wenn sich alle Beteiligten völlig rational verhalten (O'Connor \& Weatherall, 2019). ${ }^{13}$

Zwar gab es Informationskaskaden schon immer - das beste Beispiel ist die Verbreitung einfacher Gerüchte (Sunstein, 2014). Doch die Digitalisierung und insbesondere die sozialen Netzwerke wirken sich zusätzlich begünstigend auf die Entstehung von Informationskaskaden aus. Das liegt einerseits an der Beschleunigung, die die Verbreitung von Informationen durch die Digitalisierung erfahren. Zum anderen können Meinungen im Netz aufgrund der voreingestellten Möglichkeiten der Meinungsbekundung (Likes, Shares etc.) mit sehr geringem Aufwand kundgetan und weithin sichtbar gemacht werden.

Informationskaskaden sind dafür verantwortlich, dass sich auch absurde und verschwörungstheoretische »Fake News« weit verbreiten können. ${ }^{14}$ Wie glaubwürdig Menschen eine Information finden, hängt nicht nur davon ab, wie gut sie in ihr vorheriges Weltbild passt. Entscheidend ist auch, wie viele ihrer Mitmenschen sie bereits für wahr halten und verbreiten. Durch

12 Siehe Coady (1992) zur Rolle von Behauptungen anderer für die Erkenntnistheorie.

13 Die Modelle von O'Connor und Weatherall zeigen, dass Informationskaskaden auch dann eintreten und dazu führen können, dass die Gruppe zu falschen Überzeugungen gelangt, wenn alle Gruppenmitglieder gute Gründe für ihr Handeln haben, keine logischen Fehler machen und keinen kognitiven Verzerrungen unterliegen.

14 »Fake News« und Verschwörungstheorien haben einige Parallelen (vor allem bezüglich der Art ihrer Verbreitung), sind jedoch grundsätzlich unterschiedliche Phänomene (Jaster \& Lanius, im Erscheinen b). Denn im Gegensatz zu »Fake News« können Verschwörungstheorien sowohl wahr sein als auch aufrichtig geglaubt werden. So ist die Watergate-Theorie eine wahre Theorie über eine tatsächliche Verschwörung, die auch von vielen als solche erkannt und mit aufrichtigen Absichten verbreitet wurde. Siehe auch Dentith (2014). 
Informationskaskaden werden Menschen umso offener für »Fake News«, je weiter diese in ihr soziales Umfeld vorgedrungen sind.

Häufig verhalten sich sogar diejenigen, die eine Meldung nicht glauben, so, als wären sie überzeugt. Verantwortlich dafür ist der Druck zur sozialen Konformität. Menschen neigen dazu, sich den in ihren sozialen Bezugsgruppen vorherrschenden Meinungen anzupassen. Es lässt sich eine Tendenz nachweisen, die eigenen Überzeugungen zu unterdrücken, um sich nicht mit einer (manchmal auch nur als solcher wahrgenommenen) Minderheitenmeinung offen dem Druck einer Gruppe auszusetzen (Asch, 1955; Cialdini \& Goldstein, 2004).

Auch durch Konformitätsdruck kann es zu einem Kaskadenphänomen kommen. Je mehr Menschen in einer Gruppe bereits ihre Zustimmung zu einer Meinung ausgedrückt haben, desto höher sind die sozialen Kosten einer abweichenden Meinungsbekundung. Das Resultat ist eine sogenannte Konformitätskaskade. Konformitätskaskaden unterscheiden sich von Informationskaskaden in einer entscheidenden Hinsicht: Während Menschen in einer Informationskaskade ihre Überzeugungen an den Überzeugungen anderer Menschen ausrichten, passen Menschen in einer Konformitätskaskade nicht ihre Überzeugungen, sondern lediglich ihr Verhalten an. Sie verhalten sich so, als würden sie ebenfalls glauben, was die anderen glauben - zum Beispiel, indem sie eine dazu passende Meinung äußern oder Inhalte teilen. Der Verbreitungsgrad von »Fake News« lässt demnach keine unmittelbaren Rückschlüsse darauf zu, wie viele Menschen die problematischen Meldungen tatsächlich für wahr halten.

Inzwischen werden vermehrt Social Bots dafür eingesetzt, »Fake News« in den sozialen Netzwerken zu verbreiten. ${ }^{15}$ Wenn eine große Zahl solcher Bots eine Meldung likt oder teilt, sieht es für die Nutzer so aus, als sei sie von einer entsprechenden Zahl von Menschen gelikt oder geteilt worden. Neben der reinen Sichtbarkeit, die für »Fake News« auf diese Weise erzielt wird, nutzt das Vorgehen von Social Bots gezielt die psychologischen Mechanismen, die zu Informations- und Konformitätskaskaden führen. Hält erst einmal eine hinreichende Zahl von vermeintlichen Menschen eine Information für wahr, bekommt die Information für alle anderen eine höhere Glaubwürdigkeit und der Druck, die Information nicht in Zweifel zu ziehen, wächst.

15 Es herrscht Uneinigkeit, wie groß der Einfluss von Social Bots bei der Verbreitung von »Fake News« tatsächlich ist (Woolley \& Howard 2019). Ihr Einsatz in sozialen Medien verwischt jedoch zudem die Konzeptualisierung von Informationsquellen (Tandoc, Lim \& Ling, 2018). 
Die Kaskadendynamiken in Echokammern führen zu Gruppenpolarisation. ${ }^{16}$ Bereits akzeptierte Überzeugungen bilden den Hintergrund, vor dem neue Informationen ausgewertet werden. In unterschiedlichen Echokammern kommen unterschiedliche Kaskaden in Gang. Die Anfangsplausibilität einer neuen Meldung unterscheidet sich daher für Nutzer unterschiedlicher Echokammern zunehmend. Mit der Zeit driften die Weltbilder in unterschiedlichen Echokammern mehr und mehr auseinander (Mutz, 2006; Sunstein, 2009).

Polarisierte Gruppen bilden eine tribalistische Epistemologie aus (Seemann, 2017): Der Wert der Gruppenzugehörigkeit trumpft in polarisierten Gruppen den Wert der Wahrheit. Reaktionen auf zugespitzte Meldungen und insbesondere auf »Fake News« bieten den Nutzern willkommene Gelegenheiten, sich sozial zu positionieren (Lewandowsky et al., 2017). Jede geteilte Meldung, die das Weltbild der Gruppe bestätigt, entspricht in diesen Zusammenhängen einem Stammesabzeichen, das die Zugehörigkeit zur Gruppe festigt.

Da sich dieses Verhalten insbesondere in den sozialen Medien beobachten lässt, können wir von einem »digitalen Tribalismus« (Seemann, 2017) sprechen, der sich im Internet häufig konfrontativ entlädt. ${ }^{17}$ Negative Emotionen können effektiv in der Gruppe gebündelt und gegen die andere Seite kanalisiert werden: Wir gegen die - polarisierende »Fake News« funktionieren besonders gut, weil sie Bestätigungsfehler, Vertrautheitsbias und Negativitätsbias ansprechen und in tribalistischen Online-Gruppen durch Kaskadenphänomene virale Verbreitung finden.

\section{Wie sich »Fake News« auf die Politik auswirken}

Die meisten »Fake News« tauchen zunächst in Nischenforen und auf speziellen Webseiten auf und verbreiten sich von dort aus in die sozialen Medien. Ab einer entsprechenden Reichweite in den sozialen Medien werden sie auch von den klassischen Medien aufgegriffen und in die Politik getragen. ${ }^{18}$ Diese Dynamik wird auch gezielt für politische Zwecke genutzt. In

16 Siehe Myers \& Lamm (1976), Isenberg (1986) und Sunstein (2002). Siehe auch Hendricks \& Hansen (2016).

17 Für eine aufschlussreiche Studie zur Verbreitung von Hate Speech in den sozialen Medien siehe Mondal et al. (2017).

18 Die durch das Internet resultierende schnellere Taktung und höhere Gereiztheit der klassischen Medien überträgt sich ebenfalls auf die Politik. Siehe Pörksen \& Detel (2012). Diese Tatsache spielt der erfolgreichen Verbreitung von »Fake News« und ihrem Einfluss auf die Politik ebenfalls in die Hände. 
diesem Fall werden »Fake News« als Propaganda eingesetzt. ${ }^{19}$ Unter Propaganda verstehen wir Maletzke (1963) folgend den Versuch, durch Kommunikation die Meinungen, Einstellungen und Verhaltensweisen bestimmter Gruppen unter einer politischen Zielsetzung zu beeinflussen. ${ }^{20} \mathrm{Ge}-$ schieht dieser Versuch unter Zuhilfenahme von »Fake News«, können wir von $»$ Fake News «-Propaganda sprechen.

$»$ Fake News«-Propaganda kann vier grundlegende Funktionen erfüllen, die wir im Folgenden beleuchten werden:

(1) Täuschung der Öffentlichkeit

(2) Stärkung einer Gruppenidentität

(3) Demonstration von Macht

(4) Destabilisierung der politischen oder öffentlichen Ordnung

\section{(1) Täuschung der Öffentlichkeit}

Die offensichtlichste Funktion, die »Fake News« haben können, ist die Täuschung der Öffentlichkeit über politische Tatsachen. Beispielsweise verbreitete Erika Steinbach im Nachgang der sexuellen Übergriffe in der Silvesternacht 2015/16 in Köln einen Facebook-Post mit einem angeblichen Zitat der Grünen-Politikerin Claudia Roth zu den Vorfällen (Bender, 2017). Demzufolge habe Roth die Übergriffe mit den Worten kommentiert: »Wir sollten uns stärker an islamischen Werten orientieren. Der Koran bietet die Lösungsansätze, die wir brauchen, um sexuelle Übergriffe auf Frauen effektiv zu unterbinden. « Dieses falsche Zitat ist ein klarer Fall von »Fake News«: Zu Täuschungszwecken (Mangel an Wahrhaftigkeit) wird ein falsches Bild der Wirklichkeit vermittelt (Mangel an Wahrheit).

»Fake News«, die etwas Falsches behaupten und verbreitet werden, um die Öffentlichkeit zu täuschen, fallen unter den Begriff der Lüge. Wir verstehen Lügen als Behauptungen, die die Sprecherin erstens für falsch hält, und mit denen sie zweitens beabsichtigt, ihr Gegenüber zu täuschen. ${ }^{21}$ In der Literatur besteht Uneinigkeit darüber, ob es für eine Lüge genügt, wenn die Sprecherin die Behauptung für falsch hält, oder ob die Behauptung auch tatsächlich falsch sein muss (Carson, 2006). Im Zusammenhang mit »Fake News« ist dieser Punkt allerdings nicht von zentraler Bedeutung. Falsche

19 Dass »Fake News « und Propaganda zwar zusammenfallen können, aber nicht dasselbe Phänomen bezeichnen, zeigen wir in Jaster \& Lanius (Im Erscheinen a)

20 Siehe auch Jowett \& O'Donnell (2006).

$21 \mathrm{Ob}$ eine Täuschungsabsicht bestehen muss, ist umstritten. Siehe beispielsweise Fallis (2015). 
»Fake News«, die zu Täuschungszwecken eingesetzt und somit von der Sprecherin für falsch gehalten werden, sind beiden Auffassungen zufolge Lügen. ${ }^{22}$

Wird »Fake News«-Propaganda zu Täuschungszwecken eingesetzt, ist die Täuschung der Öffentlichkeit in aller Regel kein Selbstzweck. Auch die private Lüge hat selten zum alleinigen Ziel, eine falsche Überzeugung im Gegenüber hervorzurufen. ${ }^{23}$ In der Regel geht es darum, ein übergeordnetes Ziel zu erreichen. Bei »Fake News«-Propaganda ist dieses Ziel politischer oder weltanschaulicher Natur. Im Fall des falschen Zitats etwa besteht der Zweck der Täuschung in der Diskreditierung des politischen Gegners, also in diesem Fall Claudia Roths, und, pars pro toto, der Grünen insgesamt.

Verbreiten »Fake News« Lügen über konkrete Personen, können sie in Deutschland abhängig vom konkreten Sachverhalt unter den Straftatbestand der Verleumdung ( $§ 187$ StGB) oder der üblen Nachrede ( $\$ 186$ StGB) fallen. Das gilt aber nicht für jeden Fall, in dem »Fake News«-Propaganda zu Täuschungszwecken eingesetzt wird. Lügen über die Anzahl der von Migranten verübten Sexualstraftaten zum Beispiel fallen nicht klarerweise unter einen gängigen Straftatbestand.

Erhellend ist in diesem Zusammenhang die Abgrenzung von Hate Speech. Unter Hate Speech können wir Sprechakte verstehen, die eine ganze Gruppe über Merkmale wie Hautfarbe, Herkunft, sexuelle Orientierung etc. herausgreifen und aufgrund dieser Gruppenzugehörigkeit herabsetzt. ${ }^{24}$ Die falsche Statistik hat einen ähnlichen Effekt: Einer Gruppe, die über ihre Herkunftsgeschichte herausgegriffen wird, wird fälschlicherweise zugeschrieben, in besonders hohem Maße für sexuelle Übergriffe verantwortlich zu sein. Damit wird ein Stereotyp etabliert oder gefestigt, demzufolge Migranten sexuell übergriffig seien. Doch anstelle des klarerweise diskriminierenden Sprechakts »Migranten sind Vergewaltiger« wird eine objektiv anmutende statistische Falschinformation verbreitet. »Fake News«, die Gruppen anhand erfundener Meldungen über Angehörige der

22 Bloß irreführende Aussagen - und mithin irreführende »Fake News« - sind der allgemeinen Auffassung nach keine Lügen. Siehe Saul (2012).

23 Augustinus (1952) spricht im Fall der Lüge um der bloßen Täuschung willen von sogenannten echten Lügen.

24 Die Diskussion über Hate Speech ist noch verhältnismäßig jung. Über die genaue Definition des Phänomens herrscht Uneinigkeit. Für eine hilfreiche Taxonomie unterschiedlicher Arten von Hate Speech siehe Yong (2011). 
Gruppe diskreditieren, bewegen sich somit juristisch in einem Graubereich. ${ }^{25}$

Wenn »Fake News« solche Stereotype aufgreifen, lassen sie sich besonders effektiv im Internet verbreiten. Das liegt an den Gesetzmäßigkeiten der eingangs beschriebenen Aufmerksamkeitsökonomie: Wir lesen, glauben und teilen eher Nachrichten, die negativ besetzt sind und unsere Überzeugungen bestätigen. Unsere Aufmerksamkeit wird also stark von Nachrichten eingenommen, die negative Vorurteile bestätigen (wie Hate Speech). Im Internet erzeugt der Umgang und die Weiterverbreitung solcher Hate-Spe$e c h-»$ Fake News « ein willkommenes Ventil, um negative Gefühle zu kanalisieren. Eine ganze Reihe politischer Akteure hat diesen Effekt perfektioniert. Stereotypisierende »Fake News« werden gezielt in polarisierte Echokammern und deren Kaskadendynamiken eingespeist (Benkler, Faris \& Roberts, 2018).

\section{(2) Stärkung einer Gruppenidentität}

Die Polarisierung und Fragmentierung der Medienlandschaft führt zu neuen und stärker ausgeprägten (digitalen) Gruppenidentitäten. »Fake News « sind politisch auch deshalb erfolgreich, weil das Zielpublikum aufgrund der Kaskadenphänomene in den Echokammern einer polarisierten Gesellschaft auf Richtigstellungen nicht reagiert und im Zweifel an der Gruppenidentität festhält. Diese tribalistische Verhaltensweise kann gezielt von politischen Akteuren eingesetzt werden, um die eigenen Anhänger zu mobilisieren und als Gruppe zu stärken. Metaphorisch gesprochen, dienen »Fake News« in diesem Fall als »Futter für bestätigungshungrige Stämme« (Seemann, 2017, 3).

»Fake News«, die gegen Minderheiten oder Angehörige anderer Gruppen hetzen, sind besonders geeignet für diesen Zweck, da sie ihre Zielgruppe in einem vermeintlichen Kampf gegen eine andere Gruppe eint. Es ist kein Zufall, dass »Fake News« häufig Migration, Eliten oder angebliche Gewalttaten durch Angehörige von Minderheiten aufgreifen. »Fake News« dieser Art bauen ein gemeinsames Feindbild auf und sind daher besonders

25 In Deutschland gibt es auch den Straftatbestand der Volksverhetzung ( $§ 130$ StGB), der jedoch im Fall der falschen Statistik ebenfalls nicht (klar) erfüllt ist. Die Unbestimmtheit solcher Sprechakte hat gerade für das Recht selbst eine Funktion, welche Akteure wie die AfD geschickt ausnutzen können. Siehe Lanius (2019). 
gut geeignet, die Kohäsion ihrer Zielgruppe zu stärken. Die stärkeren tribalistischen Tendenzen lassen sich zu Radikalisierungszwecken nutzen.

Paradoxerweise sorgt die Falschheit der Nachrichten typischerweise für eine besonders starke Gruppenkohäsion. Bei Verlust fundamentaler und identitätsstiftender Überzeugungen entsteht ein besonders hohes Maß an kognitiver Dissonanz. Wenn eine Gruppe kollektiv falsche Überzeugungen hat, ist es essentiell, sich innerhalb der Gruppe gegenseitig zu bestätigen. Schließlich stellen Zweifel an den falschen Überzeugungen eine direkte Bedrohung der eigenen Identität dar. Jedes Gruppenmitglied ist auf immer neue Bestätigung angewiesen, um das (individuelle und kollektive) Überzeugungssystem stabil zu halten. In diesem Sinn sind manche digitale Stämme hungrig nach Bestätigung: Sie brauchen bestätigende »Fake News«, um sich zu erhalten.

\section{(3) Demonstration von Macht}

In einer dritten Funktion können »Fake News« eingesetzt werden, um Macht zu demonstrieren. Durch die Verbreitung offenkundiger »Fake News« können Politiker signalisieren, dass sie nichts darin beschränken kann, was sie öffentlich behaupten - nicht einmal die Realität selbst. Dieser Einsatz von »Fake News« entspricht Stanleys (2018) Verständnis von Propaganda, demzufolge Propaganda im Kern bereits als ein Versuch zu herrschen zu begreifen ist. Werden »Fake News« zur Machtdemonstration eingesetzt, wird eben dieser Versuch ersichtlich: Verbreiter von »Fake News « wollen in diesen Fällen nicht durch Tatsachen - auch nicht angebliche Tatsachen - überzeugen, sondern sie beabsichtigen, die Menschen durch die offene Missachtung der Wirklichkeit zu beeindrucken und für sich einzunehmen.

»Fake News« sind in solchen Fällen keine Lügen, sondern eine Spielart so genannten Bullshits. Zur Erinnerung: Unserer Definition nach verfolgen die Verbreiter von »Fake News« entweder eine Täuschungsabsicht oder sie sind der Wahrheit gegenüber gleichgültig. Für Lügen ist die Täuschungsabsicht zentral. Ist den Verbreitern einer Nachricht die Wahrheit egal, haben wir es mit Bullshit zu tun (Frankfurt 1986). Wenn »Fake News« zum Zweck der Machtdemonstration eingesetzt werden, ist nur der letzte dieser beiden Fälle einschlägig: Schließlich geht es gerade nicht um die Täuschung der Öffentlichkeit, sondern darum, die eigene Gleichgültigkeit gegenüber der Wahrheit offen zur Schau zu stellen. Entsprechend können wir hier von demonstrativem Bullshit sprechen (Jaster \& Lanius, im Erscheinen a). 
Ein gutes Beispiel für demonstrativen Bullshit liefert Putins Kommunikation im Zuge des Ukraine-Konflikts. ${ }^{26}$ Im Jahr 2014 behauptete Putin zunächst entgegen der klar erkennbaren Tatsachen, in der Ukraine befänden sich keine russische Soldaten. Einen Monat später ließ er verlauten, es handle sich »natürlich [um] unsere Soldaten. Sie haben ganz korrekt gehandelt, sehr entschlossen und professionell «. ${ }^{27}$ Es ist offenkundig, dass Putins Ziel nicht in der tatsächlichen Täuschung der Öffentlichkeit bestanden haben kann. Vielmehr demonstriert er seinen Anhängern und der globalen Öffentlichkeit mit seiner zur Schau gestellten Gleichgültigkeit gegenüber der Wahrheit seine eigene Unangreifbarkeit. ${ }^{28}$

Dieselbe Strategie zeigt sich bei der AfD und bei Donald Trump. Beide haben in verschiedenen Kontexten ihre Verachtung für Wahrhaftigkeit als Tugend ausgedrückt und offen erklärt, dass sie sich nicht an die Wahrheit gebunden fühlen. ${ }^{29}$ Diese Haltung wird unter dem Stichwort postfaktische Politik diskutiert und geht weit über das Phänomen von »Fake News« hinaus. Polarisierte und radikalisierte Medien- und allgemein Informationsumgebungen bieten (vor allem populistischen) Politikern nicht nur die Möglichkeit, durch Falschinformationen spezifische soziale Gruppen zu mobilisieren und Macht zu demonstrieren. Soziale Gruppen können auf diese Weise auch gezielt demobilisiert und demoralisiert werden, so dass Gesellschaften selbst (noch weiter) polarisiert und destabilisiert werden.

\section{(4) Destabilisierung der politischen oder öffentlichen Ordnung}

»Fake News" destabilisieren demokratische Gesellschaften auf drei unterschiedliche Weisen, nämlich (i) durch Inhalte, die Angst und Verunsicherung schüren, (ii) durch die Schwächung der seriösen Institutionen der Erkenntnisbeschaffung und (iii) durch Normverschiebungen des politisch Sagbaren. Wir besprechen diese drei Arten destabilisierender »Fake News«-Propaganda der Reihe nach.

27 Zitiert nach Rothenberg (2015).

28 Eine sehr ähnliche kommunikative Strategie nahm Russland auch in der SkripalAffäre ein, die zwar mit Leugnung begann, aber bei der schnell klar wurde, dass Putin sich nicht ernsthaft darum kümmerte, ob herauskäme, dass Russland hinter den Morden steckt.

29 So gab der AfD-Pressesprecher Christian Lüth in einem Interview offen zu, dass es egal sei, ob eine Meldung der AfD Fake sei, solange die Message stimme. Siehe Becker (2017). Trump rühmte sich sogar offen, dass er sich Dinge einfach ausdenke. Siehe Baker (2018). 
(i) Ein Blick auf die typischen Inhalte von »Fake News« zeigt, dass sie üblicherweise besorgniserregende und auf die Katastrophe zielende Narrative bedienen. Erfundene Zahlen angeblicher Vergewaltigungen durch Geflüchtete, wie sie von der AfD immer wieder verbreitet werden, haben augenscheinlich zum Ziel, die Bevölkerung zu verunsichern und negative Einstellungen gegenüber Geflüchteten hervorzurufen. Beides spielt der rechtspopulistischen Partei in die Hände.

Trump setzt ebenfalls auf solche Inhalte, wenn er »Fake News« gegen »vergewaltigende Latinos« oder »terroristische Muslime« verbreitet. Auf dieselbe Weise verstärkt Putin (insbesondere mithilfe der russischen Staatsmedien RT und Sputnik) gezielt existierende Sorgen in Russland selbst, aber auch gerade in vielen westlichen Gesellschaften. ${ }^{30}$ Etwa indem die Angst geschürt wird, die Macht nationaler Regierungen gehe sukzessive an die EU oder andere supranationale Organisationen über. Entsprechend heißt es in einem Bericht deutscher Nachrichtendienste, der Einsatz von »Fake News« in Wahlkämpfen habe dem Ziel gedient, das Vertrauen der Bürger in die Sicherheit des Landes zu erschüttern und damit die Demokratie zu schwächen (Beuth et al., 2017).

(ii) Destabilisierende Auswirkungen haben »Fake News« auch dann, wenn sie die seriösen Institutionen der Erkenntnisbeschaffung diskreditieren. In etlichen Fällen hat Trump die Wahrhaftigkeit progressiver Medien wie der New York Times oder $C N N$ in Zweifel gezogen. Immer wieder hat er sich dafür der Verbreitung von »Fake News« bedient. Am 12. Juni 2019 tweetete Trump: »The Fake News has never been more dishonest than it is today. Thank goodness we can fight back on Social Media. Their new weapon of choice is Fake Polling, sometimes referred to as Suppression Polls (they suppress the numbers). Had it in 2016, but this is worse «. ${ }^{31}$ Trumps Tweet ist ein Beispiel für ein Phänomen, das Stanley (2018) als undermining propaganda bezeichnet: Donald Trump spricht hier legitime demokratische Ideale an, die er in demselben Sprechakt zugleich performativ untergräbt.

Mit derselben Art von undermining propaganda untergräbt eine Handvoll Konzerne seit über einem halben Jahrhundert systematisch die Glaubwürdigkeit der Wissenschaft (Conway \& Oreskes, 2012). Ihre Kampagnen betreiben sie unter Mitwirkung einer kleinen Zahl von Wissenschaftlern, um Zweifel an den Erkenntnissen und Methoden der Wissenschaft zu säen. Teilweise verfolgen sie dieses Ziel mithilfe gefälschter Forschungs-

30 Siehe Giles (2016).

31 Für den Tweet siehe https://twitter.com/realDonaldTrump/status/113878972677 1732480 . 
ergebnisse (wir können hier von Fake Science sprechen), teilweise werden Studien gezielt gefördert, die gegenläufige Evidenz (die es immer gibt) für bereits nachgewiesene kausale Zusammenhänge anhäufen. Auch mediale Akteure beteiligen sich an der Verbreitung der (angeblichen) Befunde. Ein aktuelles Beispiel liefern mediale Berichte, denen zufolge es keinen wissenschaftlichen Konsens darüber gebe, ob der menschengemachte Klimawandel existiert. Insofern diese Berichte mit einer Täuschungsabsicht oder einer Bullshit-Attitüde verbreitet werden, handelt es sich um »Fake News «.

(iii) Eine dritte Ursache für die destabilisierende Wirkung von $\gg$ Fake News « besteht in der Verschiebung von Diskursnormen. Der regelmäßige Einsatz von »Fake News« kann dazu führen, dass gar nicht mehr erwartet wird, dass Politiker sich an der Wahrheit orientieren.

Grundsätzlich passen sich unsere Erwartungen und Annahmen in einem Diskurs an die Sprechhandlungen an, die im Diskurs getätigt werden. Was auch immer in einem Diskurs geschieht, wird, wenn es kontinuierlich genug passiert, zur Norm (Lewis, 1976). Für den öffentlichen politischen Diskurs bedeutet das: Sprechhandlungen, die (regelmäßig) von Autoritäten wie den Medien oder Politikern öffentlich getätigt werden, verändern die Standards dafür, welche Sprechhandlungen im öffentlichen politischen Diskurs als angemessen gelten. Anders gewendet: Wenn einflussreiche Personen der öffentlichen Debatte offen Normen verletzen, sinkt in der Gesellschaft die Akzeptanz dieser Normen tendenziell.

Soziale Normen verlieren ihre Gültigkeit jedoch nur, wenn (als solche anerkannte) Autoritäten sie besonders deutlich verletzen. Um diese Deutlichkeit sicherzustellen, setzen Trump, Putin und die AfD die so genannte Firehose-of-Falsehood-Methode ein. Dabei wird »Fake News«-Propaganda in hoher Lautstärke und auf vielen Kanälen, schnell, kontinuierlich und immer wieder ohne Rücksicht auf Konsistenz verbreitet, so dass die Öffentlichkeit kaum mehr darauf reagieren kann (Paul \& Matthews, 2016).

Dieser offensichtliche und kontinuierliche Einsatz von »Fake News« durch prominente Teilnehmer der öffentlichen Debatte bewirkt, dass die Diskursnormen des Strebens nach Wahrheit und Wahrhaftigkeit mehr und mehr ihren Stellenwert verlieren, bis die Öffentlichkeit auch kaum oder gar nicht begründete Behauptungen als Wissen akzeptiert. ${ }^{32}$ Die Standards für die Behauptbarkeit von Sachverhalten nimmt ab. ${ }^{33}$

32 Zu den unterschiedlichen Standards für die Akzeptanz von Wissen siehe insbesondere Lewis (1996) und Blome-Tillmann (2014).

33 Der sogenannten »knowledge norm of assertion« gilt, dass nur behauptet werden darf, was gewusst wird (Williamson, 1996; Williamson, 2000, 238-269). Sinken die Standards für Wissen, sinken somit auch die Standards für Behauptbarkeit. 
Im schlechtesten Fall erreicht der Normenverfall einen Punkt, an dem es für jeden einzelnen Diskursteilnehmer irrational wird, die Normen des Strebens nach Wahrheit und Wahrhaftigkeit selbst einzuhalten. Dass dieser Zustand einen destabilisierenden Effekt auf demokratische Gesellschaften hat, erschließt sich unmittelbar: Demokratien (ob direkte oder repräsentative) benötigen eine informierte öffentliche Debatte, die durch den konstanten Austausch an Meinungen und Argumenten gewährleistet, dass politische Entscheidungen auf Tatsachen, und zwar sowohl den sachlichen Gegebenheiten als auch den sozialen Einstellungen dazu, basieren (Habermas, 2006; Landemore \& Estlund, 2018).

Durch den offenen Einsatz von »Fake News«, und insbesondere durch den Einsatz der Firehose-of-Falsehood-Methode, normalisieren Akteure wie die AfD, Putin oder Trump Verhaltensweisen, die Gleichgültigkeit oder sogar Verachtung für Wahrheit und Wahrhaftigkeit ausdrücken. Wenn sich diese Haltung im öffentlichen und politischen Raum durchsetzt - aber auch erst dann, leben wir tatsächlich im vielbeschworenen, sogenannten postfaktischen Zeitalter.

\section{Konklusion}

Wir haben gezeigt, wie sich »Fake News« verbreiten und welche Auswirkungen sie auf Politik und Öffentlichkeit haben. In der Aufmerksamkeitsökonomie lassen sich negative, vertraute und sensationalistische Nachrichten besonders gut verbreiten. Aufgrund der Tendenz, kognitive Dissonanz zu vermeiden, und dem damit einhergehenden Bestätigungsfehler selektieren sich Menschen tendenziell in Echokammern. Dort können Kaskadenphänomene besonders leicht ihren Anfang nehmen und ohne maßgebliche Korrekturen ablaufen. Im Zusammenspiel mit tribalistischen Tendenzen führen diese Prozesse zu Gruppenpolarisation. Eine Reihe politischer Akteure weiß diese Dynamiken auszunutzen. »Fake News« werden in diesem Fall als Propaganda eingesetzt.

»Fake News«-Propaganda kann unterschiedlichen Zielen dienen. Erstens kann sie eingesetzt werden, um die Öffentlichkeit zu täuschen. Falschmeldungen fallen in diesem Fall unter den Begriff der Lüge. Zweitens kann »Fake News«-Propaganda verwendet werden, um Gruppenidentitäten zu stärken. Da »Fake News« systematisch die tribalistischen Tendenzen polarisierter Gruppen bespielen, können politische Akteure »Fake News« gezielt einsetzen, um ihre Anhänger zu radikalisieren. Drittens kann »Fake News«-Propaganda genutzt werden, um Macht zu demonstrieren. Werden 
offenkundig falsche Behauptungen geäußert, um die eigene Gleichgültigkeit gegenüber der Wahrheit offen zur Schau zu stellen, können wir von demonstrativem Bullshit sprechen. Viertens, schließlich, kann »Fake News«-Propaganda die Funktion haben, die öffentliche und politische Ordnung zu destabilisieren. Dies geschieht, indem Inhalte verbreitet werden, die Angst und Verunsicherung schüren, indem die seriösen Institutionen der Erkenntnisbeschaffung geschwächt oder Diskursnormen der Wahrheit und Wahrhaftigkeit verschoben oder ausgehebelt werden.

\section{Literatur}

Asch, Solomon (1955). Opinions and Social Pressure, Scientific American, 193(5), 3135.

Augustinus (1952). On lying. In: Deferrari, R. J. (Hrsg.), Saint Augustine: Treatises on Various Subjects. Fathers of the Church. Washington: Catholic University of America Press.

Baker, Peter (2018). Trump and the Truth: A President Tests His Own Credibility. The New York Times, 17. März 2018. Abgerufen von https://www. nytimes.com/2018/03/17/us/politics/trump-truth-lies.html.

Bakshy, Eytan; Messing, Solomon \& Adamic, Lada A. (2015). Exposure to Ideologically Diverse News and Opinion on Facebook. Science, 348(6239), 1130-1132. DOI: 10.1126/science.aaa1160.

Baumeister, Roy et al. (2001). Bad Is Stronger Than Good. Review of General Psychology, 5(4), 323-370. DOI: 10.1037//1089-2680.5.4.323.

Baumgaertner, Bert (2014). Yes, No, Maybe So: A Veritistic Approach to Echo Chambers Using a Trichotomous Belief Model. Synthese, 191(11), 2549-2569. DOI: 10.1007/s11220-014-04-0439-9.

Becker, Kristin (2017). AfD teilt falsches Antifa-Foto. Fake? Egal! »Es geht um die Message«. Abgerufen von https://web.archive.org/web/20180330201709/http:// faktenfinder.tagesschau.de/inland/falsches-antifa-foto-101.html.

Bender, Melanie (2017). Roth mahnt Steinbach ab. Tagesschau. Abgerufen von https://www.tagesschau.de/faktenfinder/inland/falsche-zitate-social-media101.html.

Benkler, Yochai; Faris, Rob \& Roberts, Hal (2018). Network Propaganda. Manipulation, Disinformation, and Radicalization in American Politics. New York, NY: Oxford University Press.

Beuth, Patrick et al. (2017). Krieg ohne Blut. ZEIT 9/2017.

Bikhchandani, Sushil; Hirshleifer, David \& Welch, Ivo (1998). Learning from the Behavior of Others: Conformity, Fads, and Informational Cascades. Journal of Economic Perspectives, 12(3), 151-170. DOI: 10.1257/jep.12.3.151. 
Bronstein, Michael V. et al. (2018). Belief in Fake News is Associated with Delusionality, Dogmatism, Religious Fundamentalism, and Reduced Analytic Thinking. Journal of Applied Research in Memory and Cognition. DOI: 10.1016/ j.jarmac.2018.09.005.

Carson, Thomas L. (2006). The definition of lying. Noûs, 40(2), 284-306. DOI: 10.1111/j.0029-4624.2006.00610.x.

Cialdini, Robert \& Goldstein, Noah (2004). Social influence: Compliance and conformity. Annual Review of Psychology, 55, 591-621. DOI: 10.1146/annurev.psych. 55.090902.142015

Coady, C. Anthony J. (1992). Testimony: A Philosophical Study. Oxford: Clarendon Press.

Oreskes, Naomi \& Conway, Erik M. (2012). Merchants of Doubt. How a Handful of Scientists Obscured the Truth on Issues from Tobacco Smoke to Global Warming. London: Bloomsbury.

Davenport, Thomas H. \& Beck, John C. (2001). The attention economy. Understanding the New Currency of Business. Boston, MA: Harvard Business School Press.

Dentith, Matthew R. X. (2014). The Philosophy of Conspiracy Theories. Houndmills, Basingstoke: Palgrave Macmillan.

Dentith, Matthew R. X. (2017). The Problem of Fake News, Public Reason, 8(1-2), $65-$ 79.

Fallis, Don (2015). Are Bald-Faced Lies Deceptive after All? Ratio, 28(1), 81-96. DOI: 10.1111/rati.12055

Fallis, Don \& Stokke, Andreas (2017). Bullshitting, Lying, and Indifference toward Truth. Ergo, 4(10), 277-309. DOI: 10.3998/ergo.12405314.0004.010.

Festinger, Leon (2012). Theorie der Kognitiven Dissonanz, Bern.

Frankfurt, Harry G. (1986). On Bullshit. Raritan Quarterly Review, 6(2), 81-100.

Garrett, R. Kelly (2009). Echo Chambers Online? Politically Motivated Selective Exposure among Internet News Users. Journal of Computer-Mediated Communication, 14(2), 265-285. DOI: 10.1111/j.1083-6101.2009.01440.x.

Gelfert, Axel (2018). Fake News: A Definition. Informal Logic, 38(1), 84-117. DOI: 10.22329/il.v38i1.5068.

Gentzkow, Matthew \& Shapiro, Jesse M. (2011). Ideological Segregation Online and Offline. The Quarterly Journal of Economics, 126(4), 1799-1839. DOI: 10.1093/ qje/qjr044.

Giles, Keir (2016). The Next Phase of Russian Information Warfare. Abgerufen von https://www.stratcomcoe.org/next-phase-russian-information-warfare-keir-giles.

Graham, Todd (2015). Everyday Political Talk in the Internet-Based Public Sphere. In: Coleman, S. \& D. Freelon (Hrsg.), Handbook of Digital Politics (247-263). Cheltenham: Edward Elgar.

Gutschker, Thomas (2014): Der Kreml und die Wahrheit. Putins Lügen. Frankfurter Allgemeine Zeitung, 22. Juni 2014. Abgerufen von http://www.faz.net/aktuell/ politik/ausland/der-kreml-und-die-wahrheit-putins-luegen-13002460.html. 
Habermas, Jürgen (2006). Political Communication in Media Society: Does Democracy Still Enjoy an Epistemic Dimension? The Impact of Normative Theory on Empirical Research. Communication Theory, 16(4), 411-426. DOI: 10.1111/j.1468-2885.2006. 00280.x

Habgood-Coote, Joshua (2018). Stop Talking about Fake News! Inquiry: An Interdisciplinary Journal of Philosophy, 62(9-10), 1-33. DOI: 10.1080/0020174X.2018. 1508363.

Hendricks, Vincent F. \& Hansen, Pelle G. (2016). Infostorms. Why Do we Like? Explaining Individual Behavior on the Social Net. Cham: Copernicus Books.

Hilbig, Benjamin (2009). Sad Thus True. Negativity Bias in Judgments of Truth. Journal of Experimental Social Psychology, 45(4), 983-986. DOI: 10.1016/j.jesp.2009.04.012.

Isenberg, Daniel (1986). Group Polarization. A Critical Review and Meta-Analysis. Journal of personality and social psychology, 50(6), 1141-1151. DOI: 10.1037/00223514.50.6.1141.

Jaster, Romy \& Lanius, David (2018). What is Fake News? Versus, 2(127), 207-227.

Jaster, Romy \& Lanius, David (2019). Die Wahrheit schafft sich ab. Wie Fake News Politik machen. Reclam.

Jaster, Romy \& Lanius, David (Im Erscheinen a). Wenn Wahrheit wertlos wird: Bullshit als politisches Instrument. In: Waldis, M. \& M. Hubacher (Hrsg.), Politische Bildung für die »nеue» Öffentlichkeit? Veränderte Machtstrukturen einer digitalen Gesellschaft. Wiesbaden: Springer VS.

Jaster, Romy \& Lanius, David (Im Erscheinen b). Speaking of Fake News: Definitions and Dimensions. In: Floweree, A. et al. (Hrsg.), The Epistemology of Fake News. Oxford: Oxford University Press.

Jowett, Garth \& O'Donnell, Victoria (2006). Propaganda and Persuasion. Thousand Oaks, CA: Sage.

Kahan, Dan M. (2013). Ideology, Motivated Reasoning, and Cognitive Reflection: An Experimental Study. Judgment and Decision Making, 8, 407-424. DOI: 10.2139/ ssrn. 2182588

Kahneman, Daniel (2013). Thinking, Fast and Slow. London: Penguin.

Keil, Lars-Broder \& Kellerhoff, Sven F. (2017). Fake News machen Geschichte. Gerüchte und Falschmeldungen im 20. und 21. Jahrhundert. Berlin: LinksDruck Verlag.

Kepplinger, Hans M. (1998): Der Nachrichtenwert der Nachrichtenfaktoren. In: HoltzBacha, C.; H. Scherer \& N. Waldmann (Hrsg.), Wie die Medien die Welt erschaffen und wie die Menschen darin leben (19-38). Westdeutscher Verlag, Opladen/Wiesbaden.

Kunda, Ziva (1990). The Case for Motivated Reasoning. Psychological Bulletin, 108(3), 480-498. DOI: 10.1037/0033-2909.108.3.480.

Landemore, Hélène \& Estlund, David M. (2018). The Epistemic Value of Democratic Deliberation. In: Bächtiger, A. et al. (Hrsg.), The Oxford Handbook of Deliberative Democracy (113-131). Oxford: Oxford University Press. 
Lanius, David (2019). Strategic Indeterminacy in the Law. Oxford: Oxford University Press.

Lewandowsky, Stephan et al. (2017). Beyond Misinformation. Understanding and Coping with the »Post-Truth« Era. Journal of Applied Research in Memory and Cognition, 6(4), 353-369. DOI: 10.1016/j.jarmac.2017.07.008.

Lewis, David K. (1979). Scorekeeping in a Language Game. Journal of Philosophical Logic, 8, 339-359. DOI: 10.1007/BF00258436.

Lippmann, Walter (1964): Die öffentliche Meinung. München.

Maletzke, Gerhard (1963). Psychologie der Massenkommunikation. Hamburg: Verlag Hans Bredow Institut.

Mathiesen, Kay (2019). Fake News and the Limits of Freedom of Speech. In: Fox, C. \& J. Saunders (Hrsg.), Media Ethics: Free Speech and the Requirements of Democracy (161-180). Abingdon: Routledge.

Marwick, Alice E. \& Lewis, Rebecca (2017). Media Manipulation and Disinformation Online, Data \& Society. Abgerufen von https://datasociety.net/pubs/oh/DataAnd Society_MediaManipulationAndDisinformationOnline.pdf.

McIntyre, Lee C. (2018). Post-Truth. Cambridge, MA: MIT Press.

Mondal, Mainack; Silva, Leandro Araújo \& Benevenuto, Fabrício (2017). A Measurement Study of Hate Speech in Social Media. Proceedings of the 28th ACM Conference on Hypertext and Social Media, 85-94. DOI: 10.1145/3078714.3078723.

Mukerji, Nikil S. (2018). What is Fake News? Ergo, 5(35), 923-946. DOI: 10.3998/ergo.12405314.0005.035.

Mutz, Diana C. (2006). Hearing the Other Side: Deliberative Versus Participatory Democracy. Cambridge University Press.

Myers, David G. \& Lamm, Helmut (1976). The Group Polarization Phenomenon. Psychological Bulletin, 83(4), 602-627. DOI: 10.1037/0033-2909.83.4.602.

Nguyen, C. Thi (2018). Echo Chambers and Epistemic Bubbles. Episteme, 17(2), 141161. DOI: $10.1017 /$ epi.2018.32.

Nickerson, Raymond S. (1998). Confirmation Bias. A Ubiquitous Phenomenon in Many Guises. Review of General Psychology, 2(2), 175-220. DOI: 10.1037/1089-2680.2. 2.175 .

Nyhan, Brendan \& Reifler, Jason (2010). When Corrections Fail. The Persistence of Political Misperceptions. Political Behavior, 32(2), 303-330. DOI: 10.1007/s11109010-9112-2.

O'Connor, Cailin \& Weatherall, James O. (2019). The Misinformation Age. How False Beliefs Spread. New Haven: Yale University Press.

Oliver, J. Eric \& Wood, Thomas J. (2014). Conspiracy Theories and the Paranoid Style(s) of Mass Opinion. American Journal of Political Science, 58(4), 952-966. DOI: 10.1111/ajps.12084.

Oswald, Margit E. \& Grosjean, Stefan (2004). Confirmation bias. In: Pohl, R. F. (Hrsg.), Cognitive Illusions. A Handbook on Fallacies and Biases in Thinking, Judgement and Memory (79-96). London, Psychology Press. 
Paul, Christopher \& Matthews, Miriam (2016). The Russian »Firehose of Falsehood Propaganda Model. Abgerufen von https://www.rand.org/content/dam/rand/pubs/ perspectives/PE100/PE198/RAND_PE198.pdf.

Pariser, Eli (2011). The Filter Bubble. What the Internet is Hiding from You. New York: Penguin Press, 2011.

Pennycook, Gordon \& Rand, David G. (2018). Lazy, Not Biased: Susceptibility to Partisan Fake News is Better Explained by Lack of Reasoning than by Motivated Reasoning. Cognition. DOI: 10.1016/j.cognition.2018.06.011.

Pörksen, Bernhard \& Detel, Hanne (2012). Der entfesselte Skandal. Das Ende der Kontrolle im digitalen Zeitalter. Köln: von Halem.

Rini, Regina (2017). Fake News and Partisan Epistemology. Kennedy Institute of Ethics Journal, 27(2), 43-64. DOI: 10.1353/ken.2017.0025.

Rothenberg, Christian (2015). Putin, der Friedfertige. $n$ - $t v, 24$. Februar 2015. Abgerufen von https://www.n-tv.de/politik/Putin-der-Friedfertige-article14574956.html.

Saul, Jennifer M. (2012). Lying, Misleading, and What is Said. An Exploration in Philosophy of Language and in Ethics. Oxford: Oxford University Press.

Seemann, Michael (2017). Digitaler Tribalismus und Fake News. Abgerufen von http://www.ctrl-verlust.net/digitaler-tribalismus-und-fake-news/.

Simon, Herbert (1971). Designing Organizations for an Information-Rich World. In: Greenberger, M. (Hrsg.), Computers, Communications, and the Public Interest (3852). Baltimore: John Hopkins Press.

Sproule, James M. (2005). Propaganda and Democracy. The American Experience of Media and Mass Persuasion. Cambridge: Cambridge University Press.

Stanley, Jason (2018). How Fascism Works. The Politics of Us and Them. New York: Random House.

Sunstein, Cass R. (2002). The Law of Group Polarization. Journal of Political Philosophy, 10(2), 175-195. DOI: 10.1111/1467-9760.00148.

Sunstein, Cass R. (2009). Going to Extremes. How Like Minds Unite and Divide. Oxford: Oxford University Press.

Sunstein, Cass R. (2014). On Rumors. How Falsehoods Spread, Why we Believe them, and What Can De Done. Princeton: Princeton University Press.

Sunstein, Cass R. (2017). \#Republic. Divided Democracy in the Age of Social Media. Princeton, NJ: Princeton University Press.

Tandoc, Edson C.; Lim, Zheng W. \& Ling, Richard (2018). Defining »Fake News«. A Typology of Scholarly Definitions. Digital Journalism, 6(2), 137-153. DOI: 10.1080/21670811.2017.1360143.

Tversky, Amos \& Kahneman, Daniel (1974). Judgment Under Uncertainty: Heuristics and Biases. Science, New Series, 185(4157), 1124-1131. DOI: 10.1126/ science.185.4157.1124.

Wardle, Claire \& Derakhshan, Hossein (2017). Information disorder. Toward an Interdisciplinary Framework for Research and Policy Making. Strasbourg: Council of Europe report DGI. 
Wason, Peter (1968). Reasoning About a Rule. Quarterly Journal of Experimental Psychology, 20(3), 273-281. DOI: 10.1080/14640746808400161.

Williamson, Timothy (1996). »Knowing and Asserting.«Philosophical Review, 105(4), 489-523. DOI: $10.2307 / 2998423$.

Williamson, Timothy (2000). Knowledge and its Limits. Oxford: Oxford University Press.

Woolley, Samuel C. \& Howard, Philip N. (Hrsg.), (2019). Computational Propaganda. Political Parties, Politicians, and Political Manipulation on Social Media. New York, NY: Oxford University Press.

Yong, Caleb (2011). Does Freedom of Speech Include Hate Speech? Res Publica, 17, 385-403. DOI: 10.1007/s11158-011-9158-y.

Zimmermann, Fabian \& Kohring, Matthias (2018). »Fake News « als aktuelle Desinformation. Systematische Bestimmung eines heterogenen Begriffs. Medien \& Kommunikationswissenschaft, 66(4), 526-541. DOI: 10.5771/1615-634X-2018-4-526.

Zuiderveen Borgesius, Frederik et al. (2016). Should we worry about filter bubbles? Internet Policy Review. Journal on Internet Regulation, 5(1), 1-16. DOI: 10.14763/2016.1.401. 
